Boise State University

ScholarWorks

3-2015

Reflection Waveform Inversion of Ground-Penetrating Radar Data for Characterizing Thin and Ultrathin Layers of Nonaqueous

Phase Liquid Contaminants in Stratified Media

Esther Babcock

GeoTek Alaska, Inc.

John H. Bradford

Boise State University 


\title{
Reflection waveform inversion of ground-penetrating radar data for characterizing thin and ultrathin layers of nonaqueous phase liquid contaminants in stratified media
}

\author{
Esther Babcock ${ }^{1}$ and John H. Bradford ${ }^{2}$
}

\begin{abstract}
Accurately quantifying thin-layer parameters by applying a targeted reflection waveform inversion methodology to ground-penetrating radar (GPR) reflection data may provide a useful tool for near-surface investigation and especially for contaminated site investigation where nonaqueous phase liquid (NAPL) contaminants are present. We implemented a targeted reflection waveform inversion algorithm to quantify thin-layer permittivity, thickness, and conductivity for NAPL thin $(\leq 1 / 2$ dominant wavelength $\lambda)$ and ultrathin $(\leq 1 / 8 \lambda)$ layers using GPR reflection data. The inversion used a nonlinear grid search with a Monte Carlo scheme to initialize starting values to find the global minimum. By taking a targeted approach using a time window around the peak amplitude of the reflection event of interest, our algorithm reduced the complexity in the inverse problem. We tested the inversion on three different synthetic data sets and four field data sets. In all testing, the inversion solved for NAPL-layer properties within $15 \%$ of the measured values. This algorithm provides a tool for site managers to prioritize remediation efforts based on quantitative assessments of contaminant quantity and location using GPR.
\end{abstract}

\section{INTRODUCTION}

Subsurface accumulation of nonaqueous phase liquid (NAPL) contaminants can degrade soil and groundwater resources and pose a significant risk to human health (Hwang et al., 2008). These classes of contaminants fall into two categories based on density: light NAPLs (LNAPLs) are less dense than water and thus rise to the top of a water column, whereas dense NAPLs (DNAPLs) sink
(Carcione et al., 2003; Luciano et al., 2010). Many NAPL-contaminated sites are the result of improper disposal of used solvents or fuels (Nellis et al., 2009; Brusseau et al., 2011). Over time, these NAPLs can migrate vertically and horizontally for long distances in the subsurface. LNAPLs can smear across the vadose-zone/saturated-zone interface due to fluctuations in the water table (Bradford and Deeds, 2006). DNAPLs can become trapped at permeability barriers as they simultaneously migrate downward and laterally in response to dominant groundwater gradients.

In both scenarios, the result is the same: NAPLs often disperse into thin layers. These thin, discrete layers of contamination pose a problem for traditional methods of detection such as borehole sampling (Illangasekare et al., 1995; Pankow and Cherry, 1996). A slew of geophysical techniques have proven valuable in detecting subsurface NAPLs, including ground-penetrating radar (GPR) and electrical resistance tomography (Samouëlian et al., 2005). Particularly, GPR has demonstrated its utility for characterizing contaminated sites in a rapid and cost-effective manner (Brewster and Annan, 1994; Orlando, 2002; Bradford and Deeds, 2006; Bradford and Wu, 2007; Luciano et al., 2010). Through careful assessment of GPR reflection data, practitioners may be able to identify zones of anomalous subsurface permittivity and correlate these anomalies with the presence of subsurface contamination (Carcione et al., 2003; Kana et al., 2013).

However, when NAPLs disperse into thin layers, the problem of reliably detecting and quantifying the contamination becomes more difficult. "Thin layers" are those layers in which the recorded reflection events from the top and bottom of the layer are not well separated in time (Widess, 1973). Depending on the source characteristics, noise, and other factors, this limiting layer thickness may be as high as three-fourths of the dominant wavelength of the signal $\lambda$ (Guha et al., 2005; Bradford and Deeds, 2006). Here, we take thin layers to be those whose thickness is $\leq 1 / 2 \lambda$ and ultrathin layers to be those with thickness of $\leq 1 / 8 \lambda$. In such cases, measuring layer thickness $(d)$ or effective dielectric permittivity $\left(\varepsilon_{\text {ef }}\right)$ using conven-

\footnotetext{
Manuscript received by the Editor 24 January 2014; revised manuscript received 15 October 2014; published online 4 February 2015.

${ }^{1}$ GeoTek Alaska, Inc., Anchorage, Alaska, USA. E-mail: estherbabcock@u.boisestate.edu.

${ }^{2}$ Boise State University, Department of Geosciences, Boise, Idaho, USA. E-mail: jbradford@boisestate.edu.

(C) 2015 Society of Exploration Geophysicists. All rights reserved.
} 
tional velocity analysis is impossible; we must turn to other techniques if we seek to quantify thin-layer parameters (Bradford et al., 2009). Other available tools for the GPR practitioner facing a thinlayer problem include attribute analysis, amplitude variation with offset (AVO) analysis, and inversions.

Attribute analysis of GPR data may improve detection of thin layers of subsurface NAPL contamination (Baker, 1998; Orlando, 2002; Bradford and Deeds, 2006; Deparis and Garambois, 2009; Bradford et al., 2010). Attributes include instantaneous phase, instantaneous frequency, and reflection strength. However, where detection is possible, quantification of layer properties remains problematic. For example, Hwang et al. (2008) use reflection strength to quantify relative, but not absolute, DNAPL volume during a controlled spill. Orlando (2002) concludes that extracting DNAPL layer thickness from reflection strength alone could be impossible. Orlando (2002) also uses changes in instantaneous phase and frequency to delineate a zone of DNAPL contamination but is unable to use those attributes to quantify layer thickness.

Previous research has revealed that inversion of GPR data may be a promising tool for thin-layer quantification. For example, Deparis and Garambois (2009) invert for the AVO characteristics of reflection GPR data with respect to frequency and phase. They conclude that a global inversion scheme may improve thin-layer characterization. Zeng et al. (2000) qualitatively correlate model AVO curves with field GPR data and predict that full-waveform inversion of GPR data may allow for quantitative analysis of thin layers.

Waveform inversion may provide a tool to quantify the physical parameters of thin layers at contaminated sites by directly inverting for the properties of subsurface layers (Zeng et al., 2000, Plessix et al., 2013). Waveform inversion methods can theoretically incorporate all the information present in the reflected waveform and thus may provide a tool to reliably and accurately quantify thinlayer parameters. Previous research has demonstrated the efficacy of this approach using GPR reflection data for a variety of subsurface problems, including detecting contaminant infiltration (Kalogeropoulos et al., 2013), measuring soil water content (Lambot et al., 2004; Tran et al., 2012), and quantifying subsurface $\varepsilon_{\text {ef }}$ and conductivity $(\sigma)$ (Klotzsche et al., 2010; Busch et al., 2012). However, problems such as the coupled nature of material properties, computing speed, and solution nonuniqueness can hinder the reliability of full-waveform inversion algorithms (Operto et al., 2013). Targeting a single reflection event of interest, e.g., a reflection from a contaminated zone, simplifies the inverse problem (e.g., Deparis and Garambois, 2010). With these advantages in mind, here we present and test a targeted reflection waveform inversion algorithm for quantifying thin $(\leq 1 / 2 \lambda)$ and ultrathin $(\leq 1 / 8 \lambda)$-layer properties using GPR reflection data. We first present the methodology by describing the forward algorithm and inversion process. Then, we apply the methodology on synthetic data sets and validate the approach using field data. Finally, we present our conclusions and topics for future research.

\section{METHODOLOGY}

\section{Forward model}

We use an established, 1D, vertical-incidence reflectivity method to generate the synthetic wavelet (Müller, 1985). The reflectivity method provides an exact solution to the wave equation for an electromagnetic plane wave propagating through a homogeneous, isotropic, 1D layered material. Given a layered earth model and our assumptions, the $1 \mathrm{D}$ reflectivity algorithm starts at the lowermost layer and recursively computes reflection and transmission coefficients upward through a stacked multilayer system to compute $R_{1}$, the reflectivity from the total stack which we observe at the uppermost boundary (Müller, 1985). The reflection and transmission coefficients depend on the material properties, namely, $\varepsilon_{\text {ef }}, \sigma$, and $d$ for each layer (Bradford and Deeds, 2006). The reflectivity algorithm accurately simulates scattering dispersion in thinly laminated media (Bradford, 2007). Note that although our forward algorithm can incorporate frequency-dependent permittivity and conductivity, in this study, we constrain the inversion to frequency-independent constitutive parameters.

After computing $R_{1}$, the next step is to generate a simulated reflection waveform. The modeling algorithm convolves $R_{1}$ with a user-defined source. The resulting waveform, $w_{\text {calc }}$, simulates the measured GPR signal including all multiples given the above approximations and assumptions. We use a Gabor wavelet $(G)$ as the source function. Our observations and testing demonstrate that the Gabor wavelet provides a source spectrum that approximates the source wavelet of our commercial radar system (Sensors and Software, Inc., pulseEKKO PRO) under a variety of different acquisition conditions and at different frequencies. Using Gabor functions also provides flexibility in reproducing a range of source wavelets (Morlet et al., 1982). These wavelets are the product of a Gaussian window with a sine function. The value of $G$ is defined in the frequency domain as follows:

$$
G(f)=\frac{1}{\sqrt{2 p}} e^{-\frac{2 \pi\left(f-f_{0}\right)^{2}}{4 p}} e^{-i \eta},
$$

where $f_{0}$ is the central source frequency, $\eta$ is the phase rotation, and $p$ is a function of the width of the Gabor function $\delta_{0}$ :

$$
p=\frac{1}{2 \delta_{0}^{2}} .
$$

In taking this simplified approach, we assume that a $1 \mathrm{D}$ response is an adequate representation of the radar data and that the reflections occur in the far-field. Additionally, the forward algorithm contains zero source-receiver offset. Furthermore, based on the derivation of the $1 \mathrm{D}$ reflectivity model, we are assuming that the electric properties of the individual layers are homogeneous and isotropic.

Although these assumptions are often violated, the 1D formulation provides advantages, mainly in terms of computing time. For example, using the 1D algorithm decreases computing time, and our testing (not presented here) showed that synthetic data produced using the 1D assumption were a close approximation of spreading-corrected simulations from a 2D finite difference time domain (FDTD) forward algorithm for a range of homogeneous, isotropic, flat-lying, layered earth models. Using the plane-wave solution for the 1D forward algorithm means that reflections from layer boundaries within one to three wavelengths of the surface may violate the far-field condition and negatively impact the inversion. Finally, earth properties are rarely homogeneous or isotropic. Nevertheless, assuming homogeneous and isotropic materials simplifies the forward algorithm, reduces computing time, and provides a useful, commonly used approximation for subsurface simulations. 


\section{Inversion}

The 1D forward algorithm can include any number of layers. The total set of inversion parameters used to generate $w_{\text {calc }}$ is the source wavelet parameters $\left(f_{0}, \delta_{0}\right.$, and $\left.\eta\right)$ and all layer properties $\left(\varepsilon_{\mathrm{ef}}, \sigma_{\mathrm{ef}}\right.$, and $d$ ). Thus for the three-layer case, there are a total of 12 available parameters. We can choose to invert for the values of any subset of those parameters, and we define that subset as the inversion parameters. In most cases, we assume that other methods (e.g., velocity analysis) or independent measurements (e.g., conductivity probes, direct sampling) have provided estimates of the overburden and substratum properties, and we fix the layer properties above and below the thin layer to correspond to those estimates. We then solve for thin-layer parameters as well as overburden thickness $(\ell)$.

We invert for the effective source parameters using a reflection event from an uncontaminated area that originates at the overburden/substratum interface at approximately the same depth as the target of interest. We hold the six-layer properties constant in the forward algorithm and invert for $f_{0}, \delta_{0}$, and $\eta$. Inverting for the effective source function allows the algorithm to compensate for propagation effects due to overburden characteristics. However, by taking this approach, we assume that the background electric properties are laterally homogeneous between the source calibration area and the target area. Many contaminated sites likely violate this assumption. Thus, practitioners must carefully consider this caveat and its implications when determining if the approach is applicable at a given site, as well as when interpreting the results.

The inversion uses a MATLAB-based, Nelder-Mead, gradientbased simplex search method to minimize the cost function $\varphi$ with respect to user-defined parameters as follows (Lagarias et al., 1998):

$$
\varphi=\sum\left(w_{\mathrm{obs}}-w_{\mathrm{calc}}\right)^{2}
$$

where $w_{\text {obs }}$ is the recorded wavelet and $w_{\text {calc }}$ is the calculated synthetic wavelet.

The inversion algorithm initializes by randomly select starting values using a Monte Carlo approach from a predetermined uniform distribution that bounds the range that is physically realistic for each case. The algorithm searches for sets of parameters that minimize equation 3 . The inversion continues the gradientbased search to minimize $\varphi$ with respect to the specified inversion parameters until reaching a user-specified minimum value for $\varphi$ or a userspecified number of maximum function evaluations. It returns parameters that correspond to local minimum values $\left(\varphi_{\mathrm{LM}}\right)$. Our inversion begins with 1000 random selections of starting parameters and then from each of these starting points searches for a local minimum. The user could choose to perform more than 1000 iterations of the inversion, but we do not recommend using less than 1000 . We assume that the smallest misfit from all 1000 searches represents the global minimum $\left(\varphi_{\mathrm{GM}}\right)$. The inversion solution for each userdefined inversion parameter corresponds to the arithmetic mean of parameter solutions having $\varphi_{\mathrm{GM}}$.

We estimate uncertainty from the root-meansquare error of 10,000 parameter pairs around the parameter pair corresponding to $\varphi_{\mathrm{GM}}$ and choose those paired parameters that fit equation 1 within the estimated level of noise. For the source parameters, we test the coupled uncertainty for $f_{0}, \delta_{0}$ pairs and for $f_{0}, \eta$ pairs. For the thin-layer problems, we report uncertainty from $\varepsilon_{r}, d$ pairs and from $\varepsilon_{r}, \sigma$ pairs. Although additional exploration of the solution space is necessary to fully constrain the coupled, multidimensional uncertainties, this approach gives a good idea of solution uncertainty while remaining easy to visualize.

In summary, steps for setup of the forward model and subsequent implementation of this inversion algorithm are as follows:

1) Estimate/define layer properties for contaminated and uncontaminated case.

2) Estimate/define source properties.

3) Invert for effective source wavelet parameters using an uncontaminated reflection event.

4) Define a target window around the thin-layer reflection event.

5) Define inverted parameters (usually contaminated layer properties) and invert for those parameters using the effective source function in the 1D reflectivity model.

6) Run the inversion algorithm to estimate the value of the inversion parameters using the targeted reflection event.

7) Estimate uncertainty in the inversion solution using parameter pairs.

\section{TESTING}

\section{Synthetic testing}

To test sensitivity to various permittivity configurations, we evaluate the inversion algorithm with three examples that consider common types of thin-layer contrasts, where the subscript refers to the layer number: (1) $\varepsilon_{1}<\varepsilon_{2}<\varepsilon_{3}$, (2) $\varepsilon_{1}>\varepsilon_{2}<\varepsilon_{3}$, and (3) $\varepsilon_{1}<$ $\varepsilon_{2}>\varepsilon_{3}$. We use the same 1D forward algorithm as for the inversion, and therefore there is no model error. Each synthetic example has three layers (Table 1). Each example has a different source frequency, but we also present the results in terms of the ratio of the dominant wavelength to layer thickness. We add 5\% random Gaussian noise to each synthetic trace before the inversion. We adapted

Table 1. Synthetic examples: Example 1 simulates an oil layer overlying sea water, example 2 represents a DNAPL trapped at a saturated sand/clay interface, and example 3 describes an overland flow model with a saturated sand layer underneath a dry sand overlying bedrock. Note that we generated model 1 for two layer thicknesses.

\begin{tabular}{lcccc} 
Example & Layer no. & $\varepsilon_{r}$ & $\sigma_{\text {ef }}(\mathrm{S} / \mathrm{m})$ & $d(\mathrm{~m})$ \\
\hline $\begin{array}{l}\text { (1) } f_{0}=1500 \mathrm{MHz} \\
\delta_{0}=0.3 \mathrm{~ns}\end{array}$ & 1, air & 1 & 0 & 1 \\
& 2, oil & 3.5 & $5.3 \times 10^{-4}$ & (a) $0.01(10 \% \lambda)$ \\
& 3, salt water & 81 & 1 & (b) $0.025(25 \% \lambda)$ \\
$(2) f_{0}=500 \mathrm{MHz}$ & 1, saturated sand & 22 & 0.004 & 1 \\
$\delta_{0}=0.9 \mathrm{~ns}$ & 2, DNAPL-saturated sand & 7 & $9.6 \times 10^{-4}$ & $0.02(9 \% \lambda)$ \\
& 3, clay & 35 & 0.1 & 1 \\
(3) $f_{0}=1000 \mathrm{MHz}$ & 1, dry sand & 4 & $10^{-4}$ & 1 \\
$\delta_{0}=0.5 \mathrm{~ns}$ & 2, saturated sand & 22 & 0.01 & $0.005(8 \% \lambda)$ \\
& 3, granite & 5 & $10^{-5}$ & 1 \\
\hline
\end{tabular}


each model to provide an uncontaminated case to derive the source parameters. We use these "uncontaminated" traces to invert for the effective source parameters and then proceed with the inversion for the properties of the simulated contaminant layer. We estimate uncertainties from the range of coupled parameter pairs that fit the data within $5 \%$ of $\varphi_{\mathrm{GM}}$.

\section{Synthetic examples}

Example 1 simulates an oil layer overlying seawater with $1 \mathrm{~m}$ of air between the antennas and the oil layer (Table 1). We use representative values for $\varepsilon_{r}$ and $\sigma_{\text {ef }}$ of air, oil, and saltwater, where $\varepsilon_{r}$ equals $\varepsilon_{\text {ef }}$ divided by the permittivity of free space $\varepsilon_{0}$. For this example, we generate two separate synthetic traces with oil layer thicknesses equal to $10 \% \lambda$ and $25 \% \lambda$, respectively. The second synthetic example simulates a DNAPL contaminant trapped at a sand/ clay impermeability barrier. DNAPLs can become trapped in this way at the bottom of an aquifer or at isolated clay lenses within the aquifer. The DNAPL layer thickness in this model is $9 \% \lambda$, $f_{0}$ is $1000 \mathrm{MHz}$, and $\eta=0$ (Table 1). The final synthetic example

Table 2. Inversion solution and standard deviation for effective source parameters using the reflection from layer 1/layer 3 in an uncontaminated area for synthetic examples; true $\boldsymbol{\eta}=0$ for all model source functions.

\begin{tabular}{lccccc} 
Model & $\begin{array}{c}f_{0} \text { (true value) } \\
(\mathrm{MHz})\end{array}$ & $f_{0}$ (solution) \\
$(\mathrm{MHz})$ & $\begin{array}{c}\delta_{0} \text { (true value) } \\
(\mathrm{ns})\end{array}$ & $\begin{array}{c}\delta_{0} \\
\text { (solution) }(\mathrm{ns})\end{array}$ & $\eta \times 10^{-3}$ \\
\hline 1 & 1500 & $1499 \pm 3$ & 0.3 & $0.30 \pm 0.03$ & $3.4 \pm 0.2$ \\
2 & 500 & $499 \pm 3$ & 0.9 & $0.90 \pm 0.03$ & $1.1 \pm 0.2$ \\
3 & 1000 & $1000 \pm 4$ & 0.9 & $0.89 \pm 0.04$ & $1.2 \pm 0.3$ \\
\hline
\end{tabular}

Table 3. Ultra-thin-layer parameters for synthetic testing: (a) example 1, (b) example 2, and (c) example 3 and the inversion mean calculated from all results for $\varphi_{\mathrm{GM}}$. Uncertainties for $\varepsilon_{r}, d$ pairs are in Figure 1.

\begin{tabular}{|c|c|c|c|}
\hline Parameter & True value & Solution & Bounds \\
\hline \multicolumn{4}{|l|}{ (a) } \\
\hline$\varepsilon_{e f}$ & 3.5 & (a) 3.24 (b) 3.45 & $1-50$ \\
\hline$d(\mathrm{~m})$ & (a) 0.01 (b) 0.025 & (a) 0.01 (b) 0.025 & $0-1$ \\
\hline$\ell(\mathrm{m})$ & 1 & $0.99 \pm 0.011001 \pm 0.001$ & $0-10$ \\
\hline $\begin{array}{l}\sigma(\mathrm{S} / \mathrm{m}) \\
(b)\end{array}$ & $5.3 \times 10^{-4}$ & $3.8 \pm 3.1 \times 10^{-4} 1.8 \pm 1.3 \times 10^{-4}$ & $0-0.1$ \\
\hline$\varepsilon_{e f}$ & 7 & 6.9 & $1-40$ \\
\hline$d(\mathrm{~m})$ & 0.02 & 0.02 & $0-1$ \\
\hline$\ell(\mathrm{m})$ & 0.40 & $0.399 \pm 0.002$ & $0-10$ \\
\hline $\begin{array}{l}\sigma(\mathrm{S} / \mathrm{m}) \\
(c)\end{array}$ & $9.6 \times 10^{-4}$ & $1.8 \pm 1.6 \times 10^{-4}$ & $0-0.1$ \\
\hline$\varepsilon_{e f}$ & 22 & 22.51 & $1-40$ \\
\hline$d(\mathrm{~m})$ & 0.005 & 0.0048 & $0-1$ \\
\hline$\ell(\mathrm{m})$ & 1 & $1.007 \pm 0.005$ & $0-10$ \\
\hline$\sigma(\mathrm{S} / \mathrm{m})$ & 0.01 & $8.2 \pm 3.5 \times 10^{-7}$ & $0-1$ \\
\hline
\end{tabular}

\section{Synthetic parameter sensitivity testing}

We systematically test the inversion for sensitivity to conductivity using only example 3 . We generate nine different models based on example 3 with the other parameters listed in Table 1 held constant. Each model has a different thinlayer $\sigma$ starting from example 3_1 having $\sigma=10^{-0.5} \mathrm{~S} / \mathrm{m}$ to example 3_7 having $\sigma=$ $10^{-7} \mathrm{~S} / \mathrm{m}$. The inversion solutions were within $10 \%$ of the true value for the models with the four highest $\sigma$ values $\left(10^{-2}, 10^{-1.5}, 10^{-1}\right.$, and $10^{-0.5} \mathrm{~S} / \mathrm{m}$ ), but for the other five tests, the solutions for $\sigma$ vary more than five orders of magnitude from the true value (Figure 2). We conclude that there are no discernible trends in the inversion solution accuracy over the range of $\sigma$ values from $10^{-7}$ to $10^{-1.5} \mathrm{~S} / \mathrm{m}$. Reliably estimating $\sigma$ and its uncertainty may only be possible when layer $\sigma>10^{-1.5} \mathrm{~S} / \mathrm{m}$. However, the limit for $\sigma$ estimation most likely also depends on $f_{0}$ as well as the layer thickness and overburden thickness (Tsoflias and Becker, 2008). Here, we have tested $\sigma$ sensitivity using an ultrathin layer model, and it may be possible that sensitivity will increase with increasing layer thickness.

We next test the inversion sensitivity to layer thickness using variations on example 2 . With the other parameters in example 2 held constant (Table 1), we test the following values of DNAPL-layer thicknesses: $d=0.02 \mathrm{~m}(9 \% \lambda)$, $d=0.015 \mathrm{~m}(7 \% \lambda), d=0.01 \mathrm{~m}(4 \% \lambda), d=$ $0.005 \mathrm{~m}(2 \% \lambda), d=0.002 \mathrm{~m}(0.9 \% \lambda)$, and $d=$ $0.001 \mathrm{~m}(0.4 \% \lambda)$. In this case, we fix all other parameters constant and allow $d$ to be the sole inversion parameter. The inversion algorithm was accurate within $5 \%$ in retrieving ultra-thinlayer thicknesses that were much less than $10 \% \lambda$, including two tests where $d<1 \% \lambda$ (Fig- 
ure 2). This result demonstrates that reflection radar data are sensitive to extremely thin layers $(<1 \% \lambda)$.

\section{Discussion of synthetic results}

Overall, the accuracy of our inversion algorithm for recovering thin- and ultrathin-layer parameters other than $\sigma$ using GPR reflectivity data demonstrates its potential usefulness for quantitatively characterizing thin-layer parameters. The reflection waveform inversion recovers thin-layer $\varepsilon_{r}$ within $8 \%, d$ within $14 \%$, and $\ell$ within $2 \%$ of the true values for all synthetic examples with added Gaussian noise of $5 \%$, but it is insensitive to $\sigma$ values $<10^{-1.5} \mathrm{~S} / \mathrm{m}$. Qualitatively, this result is not surprising: Low conductivity values cause a minimal effect on reflectivity and because the layer thickness is much less than the skin depth, there will be little measurable attenuation effect. Therefore, changes in $\varepsilon_{\text {ef }}$ dominate the reflectivity response at low $\sigma$ values (Zeng et al., 2000). Next, we validate the inversion algorithm on field GPR data from contaminated sites.

\section{Testing on field data}

We tested the inversion on one laboratory and two field data set examples for three different NAPL scenarios. All data were collected in transverse electric mode using Sensors and Software PulseEkko PRO antennas. Example 1 is air/oil/water. Example 2 is moist sand/LNAPL-sand/saturated sand. Example 3 is air/snow/ oil/ice, where the snow layer was a thin layer and the oil layer was an ultrathin layer. This example presents a challenging test case for the inversion algorithm because we inverted for the electric properties of the snow and oil layers.

For each data example, the reflection event from an uncontaminated area provides a background wavelet that we use to invert for the effective source function. As we described in the "Methodology" section, for the source parameter inversion, the electric properties of the uncontaminated layers are known and fixed, and $f_{0}, \delta_{0}$, and $\eta$ act as the inversion parameters. For example 3 , an additional layer forced us to simultaneously invert for the source parameters as well as additional thin-layer properties. It is important to remember
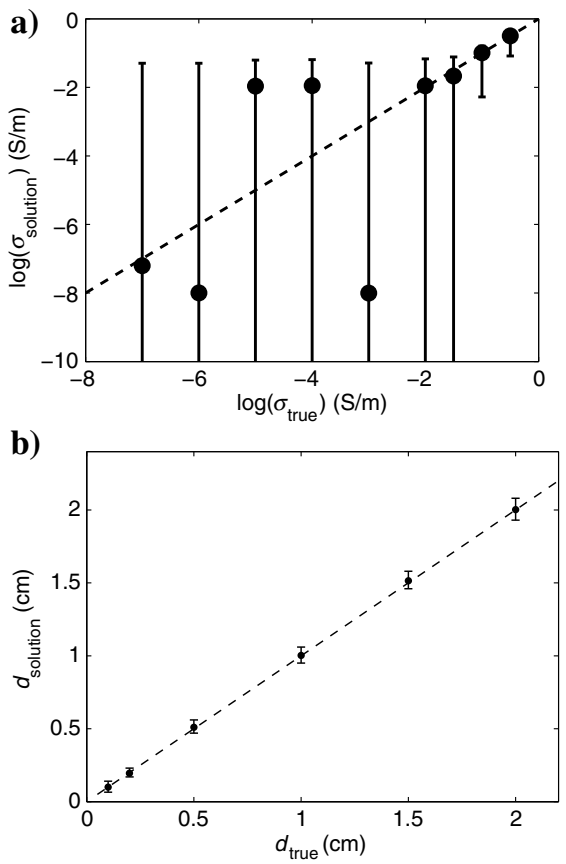

Figure 2. Synthetic sensitivity testing for (a) $\sigma$ using example 3 and (b) $d$ using example 2 , where $\cdot$ is the inversion solution, and the dashed line marks 1:1 correlation. Note scales. Error bars are those solutions within $5 \%$ of $\varphi_{\mathrm{GM}}$. The inversion does not appear to be sensitive to $\sigma$, which corroborates our observations throughout model testing. On the other hand, the inversion retrieves layer thicknesses accurately (within $5 \%$ of the true model value) down to a layer thickness of $0.4 \% \lambda$.

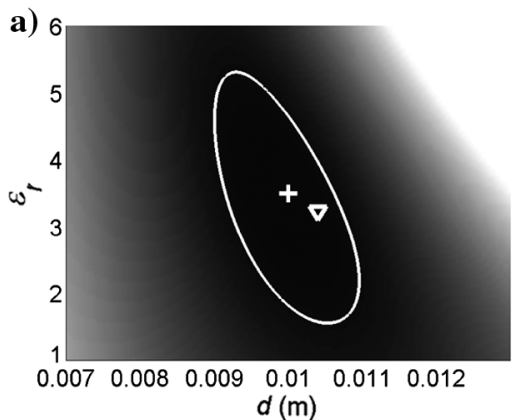

c)

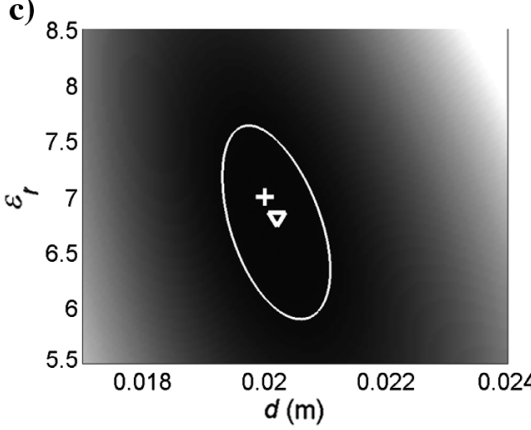

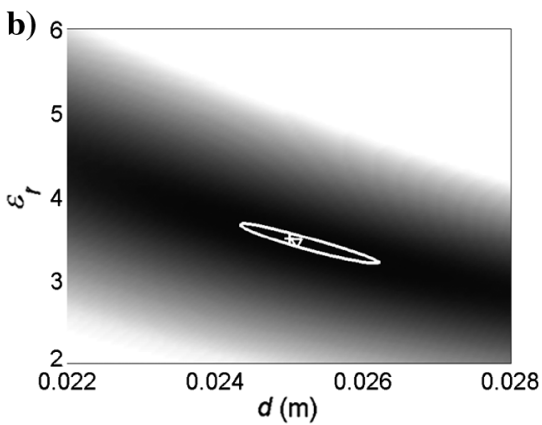

d)

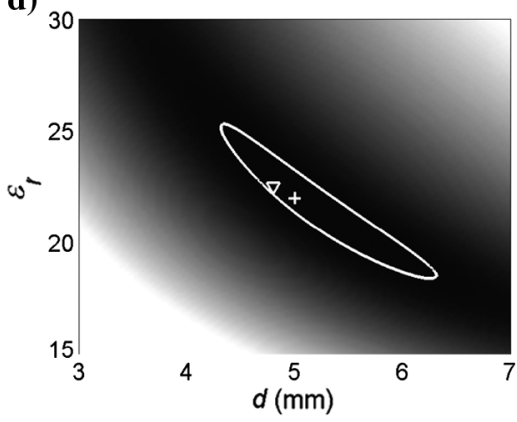

Figure 1. Synthetic testing: Uncertainties calculated for $\varepsilon_{r}, d$ pairs centered around the inversion solution for (a) example 1a, (b) example 1b, (c) example 2, and (d) example 3; darker shading indicates smaller uncertainty. The + is the exact model value, the triangle is the inversion solution, and the line encloses all paired values where the objective function is within $5 \%$ of $\varphi_{\mathrm{GM}}$. Uncertainties in solutions for $\sigma$ and $l$ are the range of values enclosed by the line for these parameters for coupled $\varepsilon_{r}, \sigma$ pairs and $\varepsilon_{r}, l$ pairs, respectively (not pictured here, values in Table 3 ). 
that in taking this approach, we assume that the background electric properties are constant over the survey area. For each example, we provide an estimated control value of the inverted parameters.

\section{Example 1}

The first data example simulates an oil spill on cold ocean water. We set up a plastic tank in a freezer room and maintained the water temperature at $2^{\circ} \mathrm{C}$ and the water salinity to 32 parts per thousand (ppt). We collected data with antennas $\left(f_{0}=1000 \mathrm{MHz}\right)$ sus- pended on a wooden plank $1.16 \mathrm{~m}$ over the water (Figure 3). After collecting control data without oil present, 189 liters of motor oil released into the tank formed a $0.027 \mathrm{~m}(16 \% \lambda)$ layer of oil over the saltwater. Addition of $0.1 \%$ by volume naphthenic acid and $0.5 \%$ by volume brine solution ( $35 \mathrm{ppt}$ ) altered the motor oil conductivity and total acid number to be more similar to that of crude oil. Direct measurements of salinity and TDR measurements for $\varepsilon_{\text {oil }}$ provided a comparative reference for inversion performance (Table 4). Preinversion data processing steps included a time-zero correction, band-pass filter (250-500-2000-4000 MHz), spherical spreading
Figure 3. Laboratory data from example 1 showing (a) diagram of setup for data collection, (b) data (solid line) from the air/salt water reflection and the inversion results (dashed line) from the source wavelet inversion, and (c) data (solid) from the air/ oil/water reflection and inversion results (dashed). Vertical dotted lines indicate the data window used for the targeted inversion algorithm. (d) Plot showing coupled uncertainties between $\varepsilon_{\mathrm{oil}}$ and $d_{\mathrm{oil}}$; the + is the solution corresponding to $\varphi_{\mathrm{GM}}$, the triangle is measured values, and the line encloses all paired values where the objective function is within $10 \%$ of $\varphi_{\mathrm{GM}}$. Darker shading indicates lower values of the objective function. a)

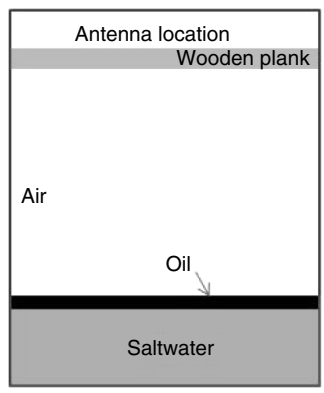

c)

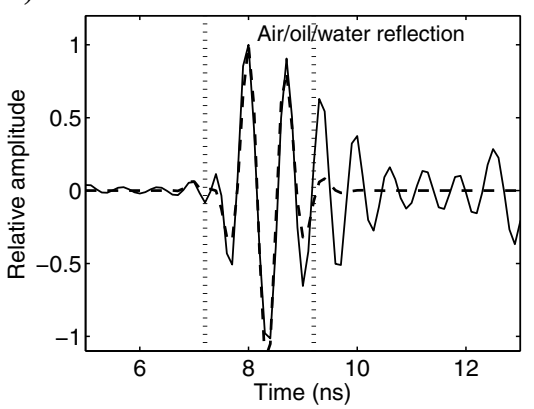

b)

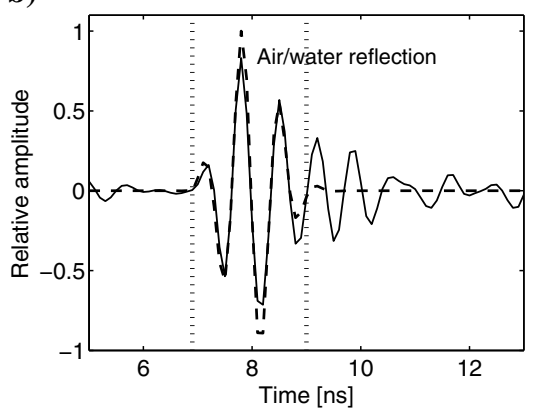

d)

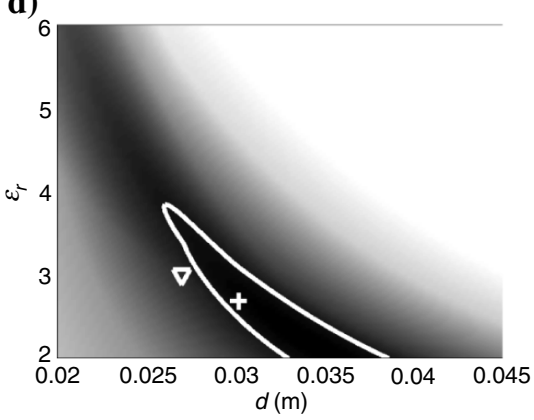

Table 4. Physical and electric properties for laboratory and field data examples.

\begin{tabular}{|c|c|c|c|c|}
\hline Data & Layer & $\varepsilon_{r}$ & $\sigma_{\mathrm{ef}}(\mathrm{S} / \mathrm{m})$ & $d(\mathrm{~m})(\% \lambda)$ \\
\hline \multirow[t]{2}{*}{ (1) $f_{0}=1000 \mathrm{MHz}$} & 1 , air & 1 & 0 & 1.01 \\
\hline & 3 , salt water & 88 & $3.5 \pm 0.1$ & 0.25 \\
\hline (2) $f_{0}=100 \mathrm{MHz}$ & 1 , dry sand & 4.9 & $5 \times 10^{-53} 2 \times 10^{-4_{3}}$ & 4 \\
\hline \multirow[t]{3}{*}{ (3a) $f_{0}=1000 \mathrm{MHz}$} & 1, air & 1 & 0 & 1 \\
\hline & 2, snow & $1.4-2$ & $10^{-5}$ & $0.05-0.14(<50 \%)$ \\
\hline & 4 , sea ice & 4.5 & 0.03 & N/A \\
\hline \multirow{2}{*}{ (3b) $f_{0}=1000 \mathrm{MHz}$} & 1, air & 1 & 0 & 1 \\
\hline & 2, snow & $1.4-2.4$ & $10^{-5_{4}}$ & $0.05-0.20$ \\
\hline
\end{tabular}

${ }^{3}$ Reported $\sigma$ values for the vadose zone are higher in contaminated region; a lower value is used in source inversion; see Sauck et al. (1998) for details.

${ }^{4}$ Estimates only; not measured on site (Bradford et al., 2010). 
correction $\left(t^{1}\right)$, and reflection event windowing (as shown for all examples by vertical dashed lines in the corresponding figures).

\section{Example 2}

The first field data example was collected at former Wurtsmith Air Force Base, Michigan. A plume of spilled LNAPL hydrocarbons is present at and just above the water table in a zone approximately $0.3 \mathrm{~m}$ thick over the vadose zone/saturated zone interface (Figure 4a). Extensive use of geophysical methods including GPR has thoroughly characterized this contaminated site (Table 4) (Bermejo et al., 1997; Sauck et al., 1998; Bradford and Deeds, 2006). Reduced reflection strength and a "shadow" zone of preferential attenuation clearly marks the contaminated region (Sauck et al., 1998; Bradford and Deeds, 2006). Bradford and Deeds (2006) estimate material properties using a calculation of the offset-dependent reflectivity and comparison of measured values to a range of background models. There are several reports in the literature for NAPL thickness at this site (Sauck et al., 1998; Bradford and Deeds, 2006).

We collected data using $100 \mathrm{MHz}$ unshielded antennas with a fixed offset of $0.3 \mathrm{~m}$ and suspended slightly above ground level. We performed the source inversion on two different traces from the uncontaminated regions (located at approximately 152 and $240 \mathrm{~m}$ ) and the thin-layer inversion using three traces from the contaminated region (at approximately 187, 198, and $210 \mathrm{~m}$ ) (Figure 4). Processing steps include a time-zero correction, band-pass filter (12-25-200-400 MHz), spherical spreading correction $\left(t^{1}\right)$, and reflection event windowing. We also tested the thin-layer inversion routine on three traces from the uncontaminated region to assess the robustness of the inversion.

\section{Example 3}

The second field example is a controlled oil spill above sea ice. Testing occurred at Svalbard, Norway. We collected radar data over clean and contaminated areas using $1000-\mathrm{MHz}$ centralfrequency antennas slung beneath a helicopter (Figure 5) (Bradford et al., 2010). In the contaminated zone, the introduced ultrathin oil layer overlying the ice was covered by a thin layer of snow. The inversion uses data from helicopter traverses at 5-m elevation above the surface; elevations are approximate due to helicopter flight characteristics. Bradford et al. (2010) provide further details on the experiment design and describe measurement of the relevant electric properties using traveltime analysis. Data processing steps included a time-zero correction, band-pass filter (250-500-2000-4000 MHz), spherical spreading correction $\left(t^{1}\right)$, background subtraction, and reflection event windowing.

For example 3, we performed the inversion routine on five different data traces: two from the uncontaminated region and three from the contaminated locations across the survey area. We hand picked data traces having different snow and oil thicknesses to demonstrate the inversion robustness. In example 3a, we used the snow/ice reflection event for the source wavelet inversion in the uncontaminated three-layer case of air/snow/ice, meaning that $f_{0}, \delta_{0}, \eta, \varepsilon_{\text {snow }}, d_{\text {snow }}$, and $\ell$ were the inversion parameters. The snow layer was less than $1 / 2 \lambda$ for all traces. For example $3 \mathrm{~b}$, two thin layers were present: snow and oil (Table 4). Thus, $\ell, \varepsilon_{\text {snow }}$, and $\varepsilon_{\text {oil }}$ as well as $d_{\text {snow }}$ and $d_{\text {oil }}$ were all inversion parameters in the contaminated area. Snow permittivity at the site varied due to wind redistribution (Bradford et al., 2010). We used the range for $\varepsilon_{\text {snow }}$ from the source inversion to bound solution values for $\varepsilon_{\text {snow }}$ in example $3 \mathrm{~b}$. The oil-layer thickness ranged from 0 to $0.036 \mathrm{~m}(<17 \% \lambda)$. The mean oil thickness was $0.0192 \mathrm{~m}$ $(<9 \% \lambda)$. We do not report values for either $\sigma_{\text {snow }}$ or $\sigma_{\text {oil }}$.

\section{Field data results}

Results for effective source parameters are in Table 5. In examples 1 and 3, with the antennas suspended in air, the inversion solution for $f_{0}$ is up to $40 \%$ greater than the manufacturer-specified $f_{0}$ (Table 5). Estimated $f_{0}$ for example 2 is within $6 \%$ of the values reported by Bradford and Deeds (2006). We estimate uncertainties for the source parameter results from coupled $f_{0}, \delta_{0}$, and $f_{0} \eta$ pairs (Table 5).

In the laboratory example, the inversion retrieves the ultrathin layers $\varepsilon_{r}$ and $d$ within $15 \%$ of the estimated control values (Figure 3 and Table 6). The inversion solution for $\sigma$ deviates over an order of magnitude from the estimated oil $\sigma$. For the Wurtsmith data, the inversion retrieves ultrathin layer $\varepsilon_{r}$ within $8 \%$ of the estimated

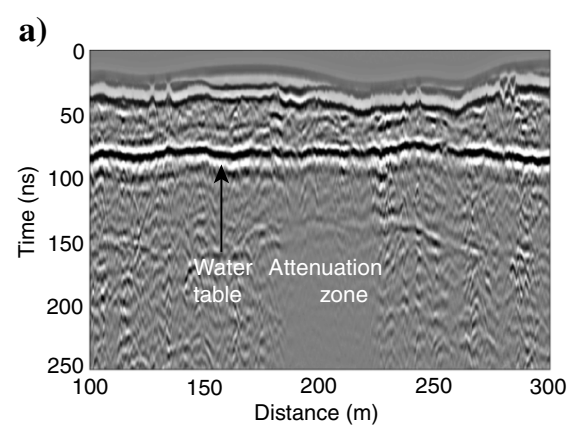

c)

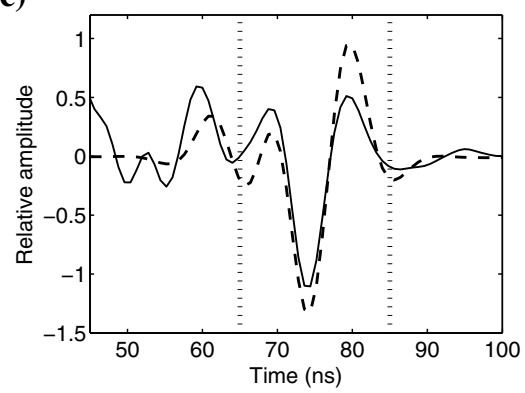

b)

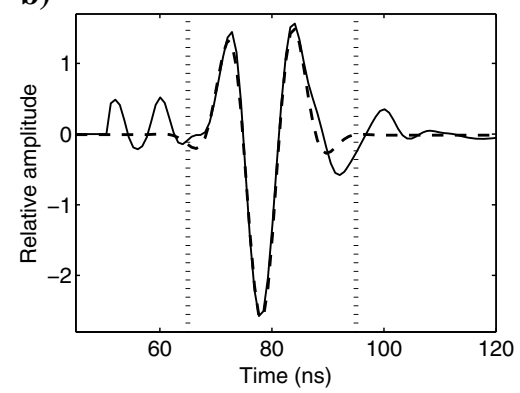

d)

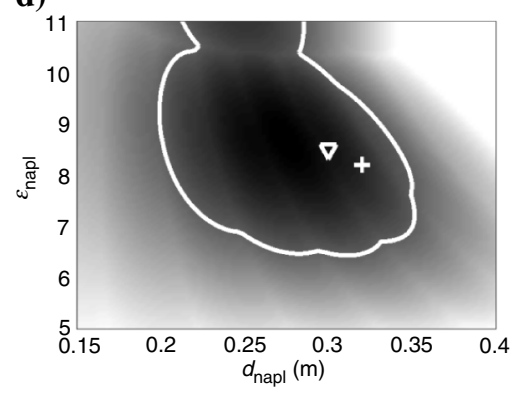

Figure 4. (a) Field data from Wurtsmith field site; the contaminated region is marked by increased attenuation below the water table. The leftmost arrow is approximate position of traces for source parameters inversion; the rightmost arrow is approximate position of traces for thin-layer inversion. (b) Data (solid line) from the uncontaminated water table reflection and the inversion results (dashed line) from the source wavelet inversion and (c) data (solid) from the LNAPL region and inversion results (dashed). Vertical dotted lines indicate the target window. (d) Coupled uncertainties between $\varepsilon_{\text {napl }}$ and $d_{\text {napl }}$; the + is the solution corresponding to $\varphi_{\mathrm{GM}}$, the triangle is measured values, and the line encloses all paired values where the objective function is within $10 \%$ of $\varphi_{\mathrm{GM}}$. Darker shading indicates lower values of the objective function. 
a)

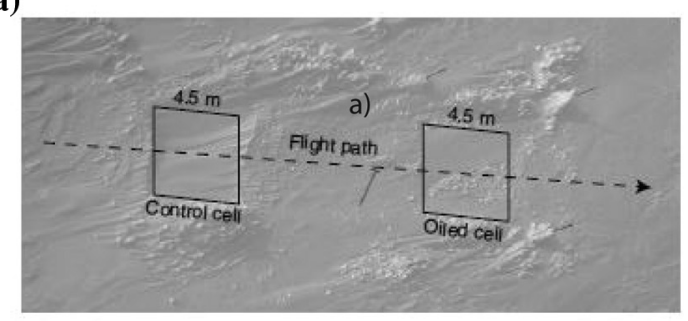

b)

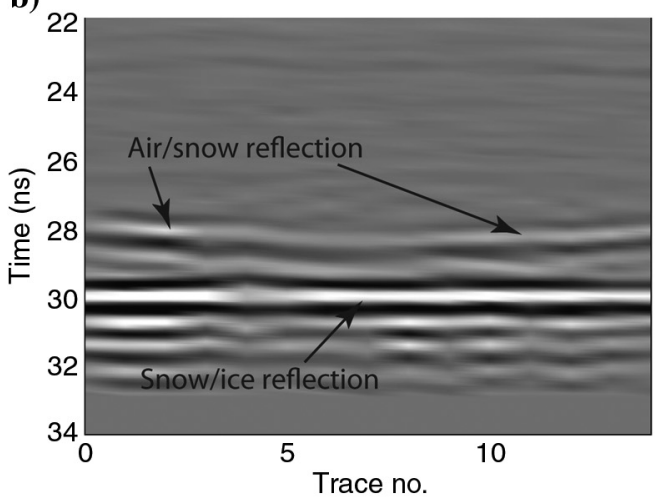

c)

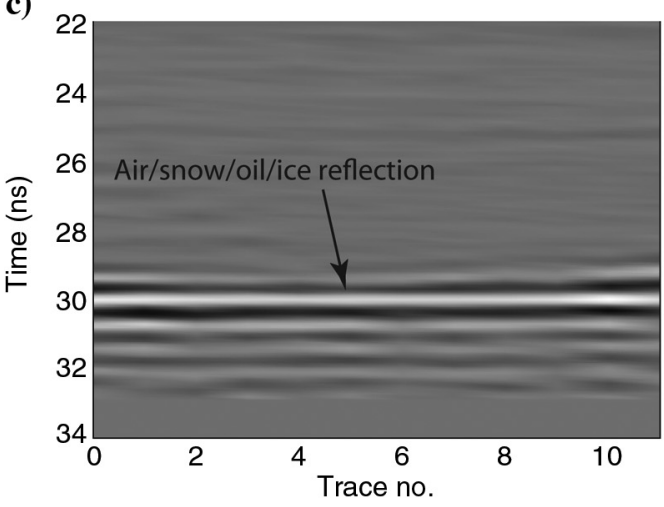

Figure 5. (a) Helicopter flight path over the uncontaminated (control) cell and the oily cell when collecting field data for example 3, (b) example data collected along the flight path over a clean test cell demonstrating variable snow thickness, and (c) oily cell (Bradford et al., 2010).

Table 5. Inversion solution and standard deviation for source wavelet parameters from field data examples using reflection from layer 1/layer 3 in an uncontaminated area; in example 3 , we simultaneously inverted for additional thin snow layer parameters; those results are in Table 6.

\begin{tabular}{lrccc} 
Example & $\begin{array}{c}\text { Source } \\
(\mathrm{MHz})\end{array}$ & $f_{0}(\mathrm{MHz})$ & $\delta_{0}(\mathrm{~ns})$ & $\eta$ \\
\hline 1 & 1000 & $1400 \pm 10$ & $0.46 \pm 0.04$ & $1.5 \pm 0.2$ \\
2 & 100 & $75 \pm 5$ & $6.3 \pm 0.7$ & $0.54 \pm 0.06$ \\
3 & 1000 & $1360 \pm 200$ & $0.63 \pm 0.08$ & $0.745 \pm 0.005$
\end{tabular}

value and $d$ within $13 \%$ (Figure 3 and Table 3 ). When tested in the uncontaminated area, the inversion retrieved the permittivity of the overburden within $2 \%\left(\varepsilon_{r}=4.8+0.2\right)$ of the value measured by Bradford and Deeds (2006) $\left(\varepsilon_{r}=4.9\right)$.

For the final field example, solutions for $\varepsilon_{\text {snow }}$ recovered during the source parameter inversion ranged from 1.46 to 1.81 (Table 6). Assuming a $d_{\text {snow }} \leq 2 \varepsilon_{0}$, these results suggest a maximum $10 \%$ error for snow permittivity in the uncontaminated area (Figure 6). Results for $d_{\text {snow }}$ during the source parameter inversion were within the measured values across the cell. In addition, the inversion solution is within the range of the data over the estimated snow layer thicknesses (Figure 6a and 6b). The inversion demonstrates an overall lack of sensitivity to $\sigma$ again in this example.

During the inversion in example $3 \mathrm{~b}$ for snow and oil thin and ultrathin layer properties, we constrained $\varepsilon_{\text {snow }}$ to the solution range from example $3 \mathrm{a}$. In this case, the inversion retrieved $\varepsilon_{\text {oil }}$ within $10 \%$ of the estimated value, and the solutions for $d_{\text {oil }}$ are within the range of measured oil thicknesses (Figure 6 and Table 6). The inversion results for $d_{\text {snow }}$ exceed the measured snow cover by a maximum of $12 \%$ (Table 6).

\section{Discussion of field data results}

In all field data examples, the inversion solved for NAPL-layer properties within $15 \%$ of the estimated control values obtained inde-

Table 6. Ultrathin-layer parameters for field examples: (a) example 1, (b) example 2, (c) example 3a, and (d) example $3 \mathrm{~b}$ with the inversion results corresponding to $\varphi_{\mathrm{GM}}$. Uncertainties for $\varepsilon_{r}, d$ pairs are shown graphically in Figures 1, 3, 6, and 7 .

\begin{tabular}{|c|c|c|c|}
\hline Parameter & Control value & Solution & Bounds \\
\hline \multicolumn{4}{|l|}{ (a) } \\
\hline$\varepsilon_{\text {oil }}$ & 3 & $2.686 \pm 1.3$ & $2-8$ \\
\hline$d_{\text {oil }}(\mathrm{m})$ & 0.027 & $0.030 \pm 0.05$ & $0-1$ \\
\hline$\ell(\mathrm{m})$ & 1.03 & $0.965 \pm 0.001$ & $0-5$ \\
\hline$\sigma(\mathrm{S} / \mathrm{m})$ & $\approx 5 \times 10^{-4}$ & $8 \pm 3 \times 10^{-6}$ & $0-0.1$ \\
\hline \multicolumn{4}{|l|}{ (b) } \\
\hline$\varepsilon_{\text {napl }}$ & 8.5 & $8.2 \pm 1.5$ & $2-12$ \\
\hline$d_{\text {napl }}(\mathrm{m})$ & 0.3 & $0.34 \pm 0.06$ & $0-1$ \\
\hline$\ell(\mathrm{m})$ & 4 & $4.1 \pm 0.2$ & $2-10$ \\
\hline$\sigma(\mathrm{S} / \mathrm{m})$ & $0.016 \pm 0.007$ & $0.001 \pm 0.001$ & $0-0.1$ \\
\hline \multicolumn{4}{|l|}{$(c)$} \\
\hline$\varepsilon_{\text {snow }}$ & $1.4-2$ & $1.46-1.81$ & $1-5$ \\
\hline$d_{\text {snow }}(\mathrm{m})$ & $0.05-0.14$ & $0.04-0.15^{5}$ & $0-1$ \\
\hline$\ell(\mathrm{m})$ & $5-10$ & $5.4-8.3$ & $5-15$ \\
\hline \multicolumn{4}{|l|}{$(d)^{6}$} \\
\hline$\varepsilon_{\text {oil }}$ & 3.5 & $3.2 \pm 0.2$ & $1-8$ \\
\hline$d_{\text {snow }}(\mathrm{m})$ & $0.04-0.07$ & $0.005-0.0787$ & $0.001-1$ \\
\hline$d_{\text {oil }}(\mathrm{m})$ & $0-0.036$ & $0.004-0.0321$ & $0.001-1$ \\
\hline$\ell(\mathrm{m})$ & $5-10$ & $8.67 \pm 0.5$ & $0-20$ \\
\hline
\end{tabular}

${ }^{5}$ Dependent on snow depth at trace location; see Figure 7.

${ }^{6}$ Snow $\sigma$ and oil $\sigma$ not measured at field location. 
pendently. Testing on the data from the Wurtsmith site demonstrated the capability of the inversion algorithm at a different frequency because we collected data using $100 \mathrm{MHz}$ antennas as opposed to examples 1 and 3 with $1000 \mathrm{MHz}$ antennas. The inversion algorithm was able to recover effective source wavelet parameters and an additional set of thin-layer parameters simultaneously in example 3 .

In example $3 \mathrm{a}$, the inversion results for $\varepsilon_{\text {snow }}$ from the source parameter inversion agreed well with the findings of Bradford et al. (2010). They observed that snow densities, and therefore permittivities, were relatively low for the loosely packed snow in the uncontaminated area, and our results for $\varepsilon_{\text {snow }}$, ranging from 1.46-1.81, are within $10 \%$ of the values measured in the field (Table 5). When inverting for snow and oil-layer properties simultaneously in example $3 b$, the agreement of our results with control data is remarkable considering the thinness of the oil and snow layers and the added difficulty of having one thin layer (snow) and one ultrathin layer (oil) present above the ice.

However, the inversion results for $\sigma$ deviate from the control values. Our synthetic testing (Figure 2) indicated that the inversion is insensitive to $\sigma$ until reaching a certain threshold value. That threshold may be $>0.03 \mathrm{~S} / \mathrm{m}$, but it would depend on a variety of factors including layer thickness. Note that none of the field data had a thin-layer $\sigma$ greater than that value. In addition, because our forward algorithm does not include spherical divergence, inaccurate spreading compensation during data processing could impact the inversion results for conductivity. Thus, we caution that this inversion algorithm, while performing robustly for $\varepsilon_{r}$ and $d$, is not likely to retrieve $\sigma$ reliably for thin layers of these types of contaminants. Continued work to retrieve thinlayer $\sigma$ should include testing on data at lower frequencies, e.g., $<100 \mathrm{MHz}$, as per Tsoflias and Becker (2008).

Finally, our reflection waveform inversion relies on a user-defined window to target the reflection event. Correctly identifying and windowing the desired reflection event is paramount for robust inversion performance. The choice of the reflection window has a large impact on inversion results and subsequent errors. Our testing indicates that choosing a shorter window length centered on the peak of the reflection event promotes more reliable inversion performance (Figure 7). In fact, this result is promising because it demonstrates that the inversion algorithm may perform robustly even in the presence of noise because it depends on more of the information within the wavelet that is contained near the peak a)

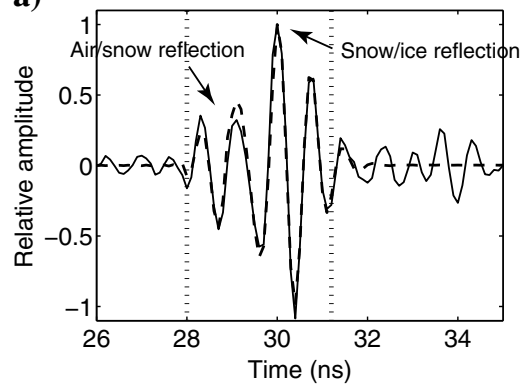

c)

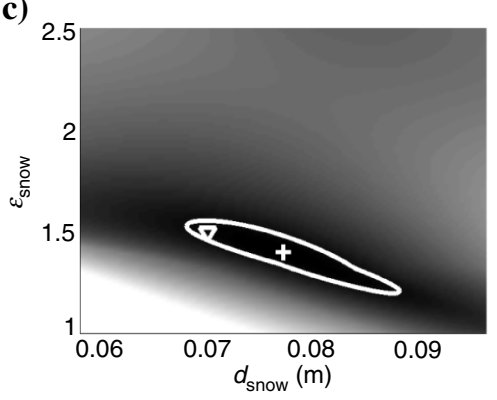

e)

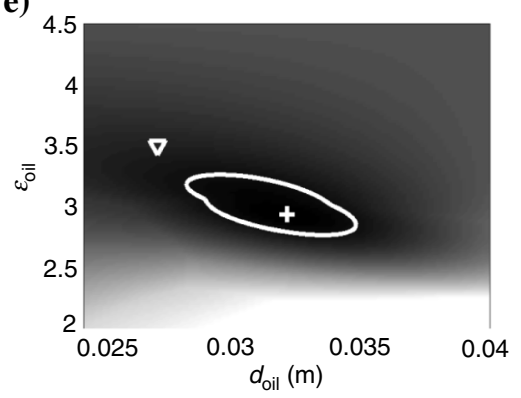

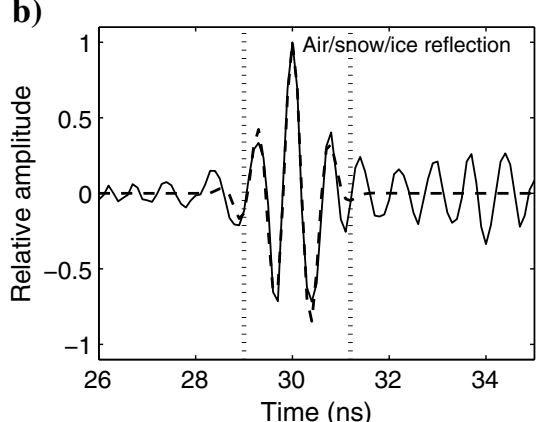

d)

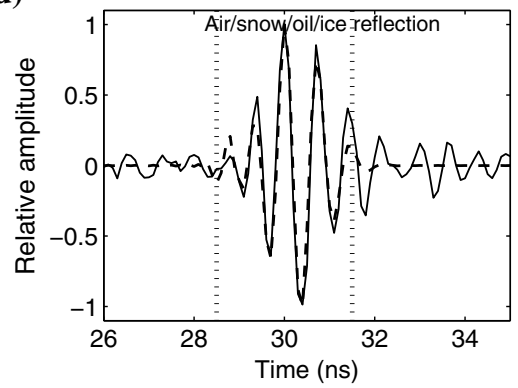

Figure 6. Field data (solid) and inversion results (dashed) for example 3. Labels indicate reflection events, and vertical dotted lines show the data window used for the targeted inversion algorithm. (a) Uncontaminated snow over ice with $d_{\text {snow }} \cong 0.11 \mathrm{~m}(46 \% \lambda)$; (b) uncontaminated snow over ice with $d_{\text {snow }} \cong 0.07 \mathrm{~m}(25 \% \lambda)$; (c) plot showing coupled uncertainties between $\varepsilon_{\text {snow }}$ and $d_{\text {snow }}$ for (b); the + is the solution corresponding to $\varphi_{\mathrm{GM}}$, the triangle marks the estimated values, and the line encloses all paired values where the objective function is within $10 \%$ of $\varphi_{\mathrm{GM}}$. (d) Data (solid) and inversion results (dashed) for an ultrathin oil layer $(0.02 \mathrm{~m}, 9 \% \lambda)$ underneath a thin snow layer $(0.04 \mathrm{~m}, 16 \% \lambda)$ (e) Plot showing coupled uncertainties between $\varepsilon_{\mathrm{oil}}$ and $d_{\mathrm{oil}}$; notation same as part [c]).

a)

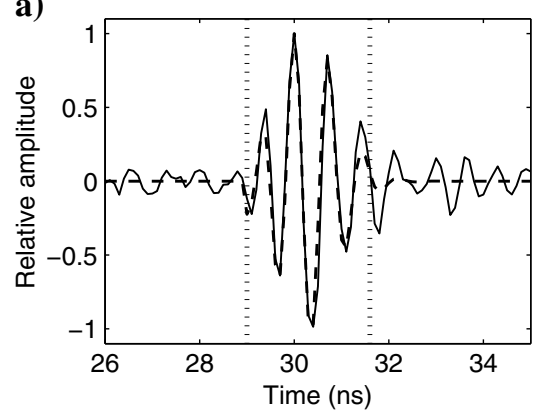

b)

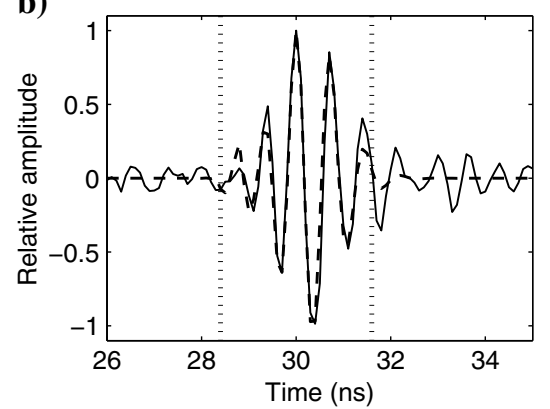

Figure 7. Inversion results (dashed) plotted versus data (solid) to demonstrate the effect of changes in the user-defined reflection window on solution accuracy; dashed lines show the data window used for the targeted inversion algorithm. (a) The solution corresponding to data windowed between 29.0 and $31.5 \mathrm{~ns}$ and (b) the solution when using a longer reflection window (28.5-31.5 ns). The solution shown in (b) returns anomalously high values for $\varepsilon_{\mathrm{oil}}(>6)$. 
of the reflection event and that peak is less sensitive to noise than the edges of the wavelet.

\section{CONCLUSIONS}

Given an effective source parameter function, the reflection waveform inversion recovered thin and ultrathin layer $\varepsilon_{r}$ and $d$ within $15 \%$ of the measured or estimated control values down to layer thicknesses as low as $9 \% \lambda$. Reliable estimation of thin-layer parameters using this inversion algorithm hinges on estimating the effective source function. Our source wavelet inversion was able to recover effective source parameters and even additional thin-layer parameters. Overall, the accuracy of our inversion algorithm for recovering thin- and ultrathin-layer parameters other than $\sigma$ using GPR reflectivity data demonstrates its potential usefulness for quantitatively characterizing contaminated sites. We caution that accurate site characterization, e.g., measuring overburden permittivity, must occur prior to inverting for thin-layer parameters. Finally, the inversion may provide reliable estimates of layer thickness well below the conventional thin-layer resolution limits, and even at layer thicknesses below $1 \% \lambda$ as demonstrated during synthetic testing.

We caution the reader that the robustness of the inversion algorithm, of course, relies on the reliability of the forward algorithm, and we make several simplifying assumptions to use the $1 \mathrm{D}$, vertical incidence, layered reflectivity model. Where these assumptions prove problematic, the user can compensate to some extent. For example, if the overburden above the contaminant is not homogeneous, but is layered, the user could easily modify the forward algorithm to include extra layers while still only inputting the contaminant-layer properties as the inversion parameters. In addition, because the 1D forward algorithm assumes vertical incidence, increasing separation between the transmitter and receiver may escalate error in the inversion results. We recommend using the inversion only with near-offset data. In fact, our method provides an alternative to equipment-intensive multioffset measurements at contaminated sites.

Our testing and observations indicate that practitioners could implement this algorithm to characterize contaminated sites at which contamination has dispersed throughout the subsurface into thin and ultrathin layers. Practitioners must carefully evaluate the lateral homogeneity of the overburden prior to applying this inversion algorithm over an entire contaminated site. Nevertheless, careful use of this inversion could reduce remediation costs and time. Because we use an effective source function inversion, the inversion is well suited for application to targeted time-lapse monitoring of contaminated sites. Time-lapse monitoring also can aid inversion performance by providing additional, ongoing information about site characteristics, e.g., overburden permittivity. Finally, the accuracy of the inversion performance for the third synthetic example suggests that this targeted waveform algorithm may be applied to other thin-layer problems such as snowmelt monitoring or fracture characterization. Additional work includes testing the inversion algorithm on more complicated field sites, such as those with varying overburden characteristics; additional testing on target window length; and parameter sensitivity testing on field data.

\section{REFERENCES}

Baker, G. S., 1998, Applying AVO analysis to GPR data: Geophysical Research Letters, 25, 397-400, doi: 10.1029/97GL03773.
Bermejo, J. L., W. A. Sauck, and E. A. Atekwana, 1997, Geophysical discovery of a new LNAPL plume at the former Wurtsmith AFB, Oscoda, Michigan: Groundwater Monitoring \& Remediation, 17, 131-137, doi: 10 .1111/j.1745-6592.1997.tb01273.x.

Bradford, J. H., 2007, Frequency-dependent attenuation analysis of groundpenetrating radar data: Geophysics, 72, no. 3, J7-J16, doi: 10.1190/1 .2710183 .

Bradford, J. H., W. P. Clement, and W. Barrash, 2009, Estimating porosity with ground-penetrating radar reflection tomography: A controlled 3-D experiment at the Boise Hydrogeophysical Research Site: Water Resources Research, 45, W00D26, doi: 10.1029/2008WR006960.

Bradford, J. H., and J. C. Deeds, 2006, Ground-penetrating radar theory and application of thin-bed offset-dependent reflectivity: Geophysics, 71, no. 3, K47-K57, doi: 10.1190/1.2194524.

Bradford, J. H., D. F. Dickins, and P. J. Brandvik, 2010, Assessing the potential to detect oil spills in and under snow using airborne ground-penetrating radar: Geophysics, 75, no. 2, G1-G12, doi: 10.1190/1.3312184.

Bradford, J. H., and Y. Wu, 2007, Instantaneous spectral analysis: Time-frequency mapping via wavelet matching with application to contaminated site characterization by 3D GPR: The Leading Edge, 26, 1018-1023, doi: 10.1190/1.2769559.

Brewster, M. L., and A. P. Annan, 1994, Ground-penetrating radar monitoring of a controlled DNAPL release: $200 \mathrm{MHz}$ radar: Geophysics, 59, 1211-1221, doi: 10.1190/1.1443679.

Brusseau, M. L., K. C. Carroll, T. Allen, J. Baker, W. DiGuiseppi, J. Hatton, C. Morrison, A. Russo, and J. Berkompas, 2011, Impact of in situ chemical oxidation on contaminant mass discharge: Linking source-zone and plume-scale characterizations of remediation performance: Environmental Science and Technology, 45, 5352-5358, doi: 10.1021/es200716s.

Busch, S., J. van der Kruk, J. Bikowski, and H. Vereecken, 2012, Quantitative conductivity and permittivity estimation using full-waveform inversion of on-ground GPR data: Geophysics, 77, no. 6, H79-H91, doi: 10 $.1190 /$ geo2012-0045.1.

Carcione, J. M., G. Seriani, and D. Gei, 2003, Acoustic and electromagnetic properties of soil saturated with salt water and NAPL: Journal of Applied Geophysics, 52, 177-191, doi: 10.1016/S0926-9851(03)00012-0.

Deparis, J., and S. Garambois, 2009, On the use of dispersive APVO GPR curves for thin-bed properties estimation: Theory and application to fracture characterization: Geophysics, $\mathbf{7 4}$, no. 1, J1-J12, doi: 10.1190/1 .3008545 .

Deparis, J., and S. Garambois, 2010, Inversion methodology of dispersive amplitude and phase versus offset of GPR curves (DAPVO) for thin beds, in R. D. Miller, J. H. Bradford, and K. Hollinger, eds., Advances in nearsurface seismology and ground-penetrating radar: SEG, 219-232.

Guha, S., S. E. Kruse, E. E. Wright, and U. E. Kruse, 2005, Spectral analysis of ground penetrating radar response to thin sedimentary layers: Geophysical Research Letters, 32, L23304, doi: 10.1029/2005GL023933.

Hwang, Y. K., A. L. Endres, S. D. Piggott, and B. L. Parker, 2008, Longterm ground penetrating radar monitoring of a small volume DNAPL release in a natural groundwater flow field: Journal of Contaminant Hydrology, 97, 1-12, doi: 10.1016/j.jconhyd.2007.11.004.

Illangasekare, T. H., J. L. Ramsey, K. H. Jensen, and M. B. Butts, 1995, Experimental study of movement and distribution of dense organic contaminants in heterogeneous aquifers: Journal of Contaminant Hydrology, 20, 1-25, doi: 10.1016/0169-7722(95)00045-W.

Kalogeropoulos, A., J. van der Kruk, J. Hugenschmidt, J. Bikowski, and E. Bruhwiler, 2013, Full-waveform GPR inversion to assess chloride gradients in concrete: NDT\&E International, 57, 74-84, doi: 10.1016/j .ndteint.2013.03.003.

Kana, A. A., L. J. West, and R. A. Clark, 2013, Fracture aperture and fill characterization in a limestone quarry using GPR thin-layer AVA analysis: Near Surface Geophysics, 11, 293-305, doi: 10.3997/1873-0604 .2012066 .

Klotzsche, A., J. van der Kruk, G. A. Meles, J. Doetsch, H. Maurer, and N. Linde, 2010, Full-waveform inversion of cross-hole ground-penetrating radar data to characterize a gravel aquifer close to the Thur River, Switzerland: Near Surface Geophysics, 8, 635-649, doi: 10.3997/1873-0604 .2010054 .

Lagarias, J. C., J. A. Reeds, M. H. Wright, and P. E. Wright, 1998, Convergence properties of the Nelder-Mead simplex method in low dimensions: SIAM Journal on Optimization, 9, 112-147, doi: 10.1137/ S1052623496303470.

Lambot, S., M. Antoine, I. van den Bosch, E. C. Slob, and M. Vanclooster, 2004, Electromagnetic inversion of GPR signals and subsequent hydrodynamic inversion to estimate effective vadose zone hydraulic properties: Vadose Zone Journal, 3, 1072-1081, doi: 10.2136/vzj2004.1072.

Luciano, A., P. Viotti, and M. P. Papini, 2010, Laboratory investigation of DNAPL migration in porous media: Journal of Hazardous Materials, 176, 1006-1017, doi: 10.1016/j.jhazmat.2009.11.141.

Morlet, J., G. Arens, E. Fourgeau, and D. Giard, 1982, Wave-propagation and sampling theory 1 . Complex signal and scattering in multilayered media: Geophysics, 47, 203-221, doi: 10.1190/1.1441328. 
Müller, G., 1985, The reflectivity method: A tutorial: Journal of Geophysics, 58, $153-174$

Nellis, S. R., H. Yoon, C. J. Werth, M. Oostrom, and A. J. Valocchi, 2009, Surface and interfacial properties of nonaqueous-phase liquid mixtures released to the subsurface at the Hanford site: Vadose Zone Journal, 8, 343-351.

Operto, S., Y. Gholami, V. Prieux, A. Ribodetti, R. Brossier, L. Metivier, and J. Virieux, 2013, A guided tour of multi-parameter full-waveform inversion with multi-component data: From theory to practice: The Leading Edge, 32, 1040-1054, doi: 10.1190/tle32091040.1.

Orlando, L., 2002, Detection and analysis of LNAPL using the instantaneous amplitude and frequency of ground-penetrating radar data: Geophysical Prospecting, 50, 27-41, doi: 10.1046/j.1365-2478.2002 .00288.x.

Pankow, J. F., and J. A. Cherry, 1996, Dense chlorinated solvents and other DNAPLs in groundwater: Waterloo Press.

Plessix, R. E., P. Milchik, H. Rynja, A. Stopin, and K. Matson, 2013, Multiparameter full-waveform inversion: Marine and land examples : The Leading Edge, 32, 1030-1038, doi: 10.1190/tle32091030.1.
Samouëlian, A., I. Cousin, A. Tabbagh, A. Bruand, and G. Richard, 2005, Electrical resistivity survey in soil science: A review: Soil and Tillage Research, 83, 173-193, doi: 10.1016/j.still.2004.10.004.

Sauck, W. A., E. A. Atekwana, and M. S. Nash, 1998, High conductivities associated with an LNAPL plume imaged by integrated geophysical techniques: Journal of Environmental \& Engineering Geophysics, 2, 203-212.

Tran, A. P., M. R. M. Ardekani, and S. Lambot, 2012, Coupling of dielectric mixing models with full-wave ground-penetrating radar signal inversion for sandy-soil-moisture estimation: Geophysics, 77, no. 3, H33-H44, doi: 10.1190/geo2011-0100.1.

Tsoflias, G. P., and M. W. Becker, 2008, Ground-penetrating radar response to fracture-fluid salinity: Why lower frequencies are favorable for resolving salinity changes: Geophysics, 73, no. 5, J25-J30, doi: 10.1190/1.2957893.

Widess, M. B., 1973, How thin is a thin bed?: Geophysics, 38, 1176-1180, doi: $10.1190 / 1.1440403$.

Zeng, X. X., G. A. McMechan, and T. Xu, 2000, Synthesis of amplitudeversus-offset variations in ground-penetrating radar data: Geophysics, $\mathbf{6 5}$ 113-125, doi: 10.1190/1.1444702. 